\title{
Inhaltsverzeichnis
}

\section{ÖKOLOGISCHE UND SOZIALE TRAGFÄHIGKEITEN ALS MANAGEMENTHERAUSFORDERUNGEN FÜR SUBURBANE BIOSPHÄRENPARKE AM BEISPIEL UNTERE LOBAU}

KURZFASSUNG

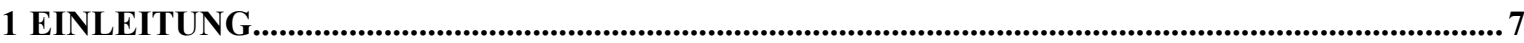

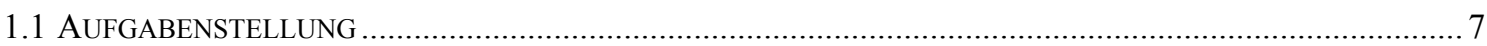

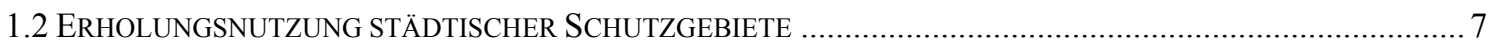

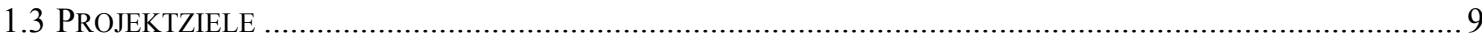

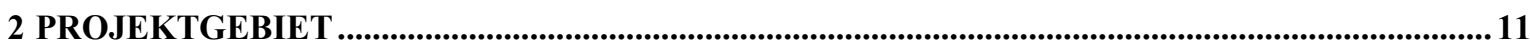

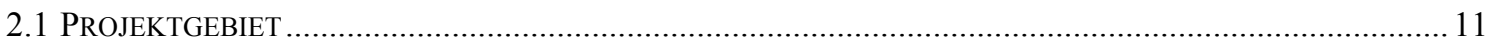

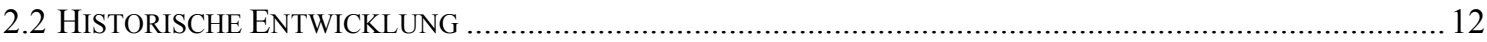

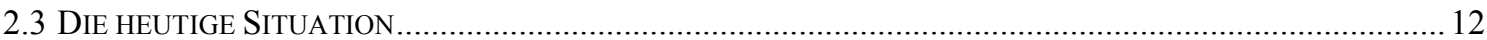

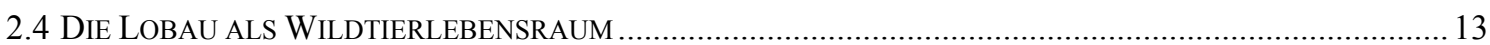

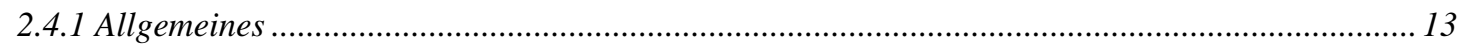

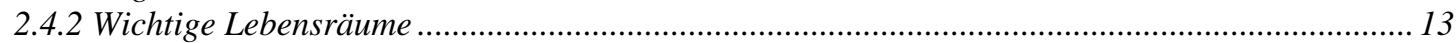

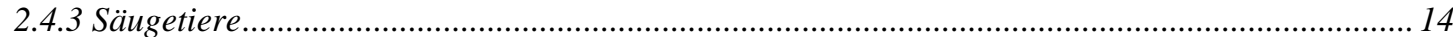

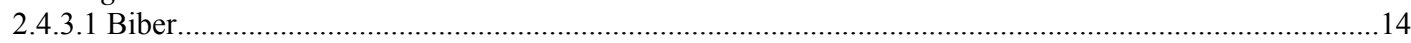

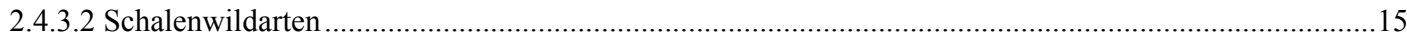

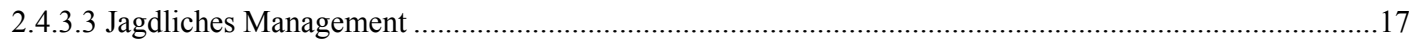

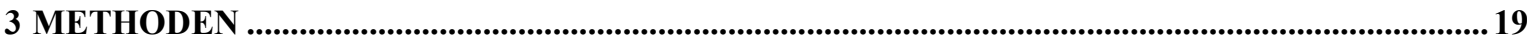

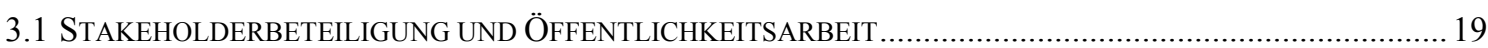

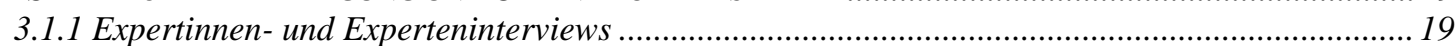

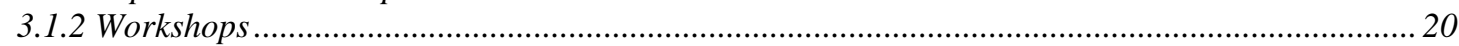

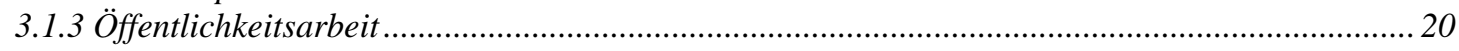

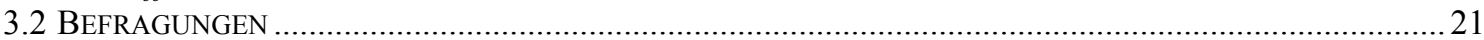

3.2.1 Postalische Befragung der lokalen Bevölkerung...................................................................2 21

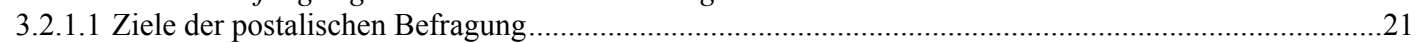

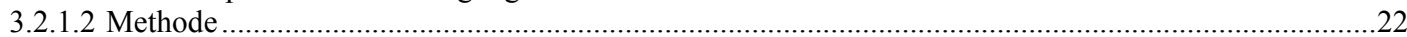

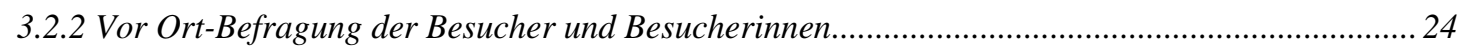

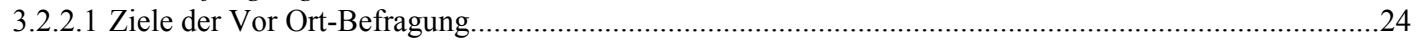

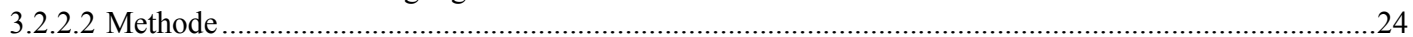

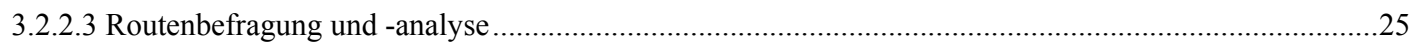

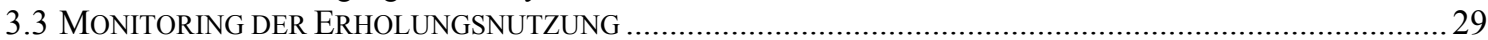

3.3.1 Bildgestützte Besucherzählung .................................................................................................... 29

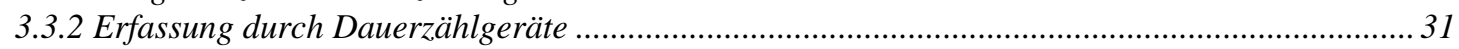

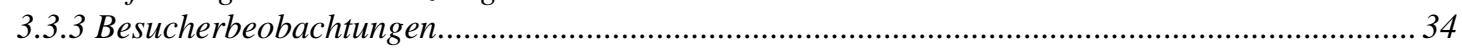

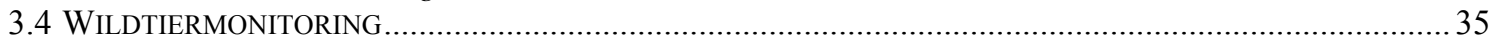

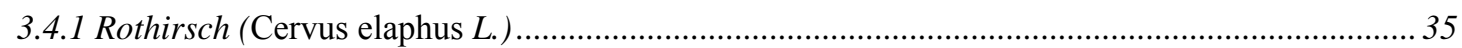

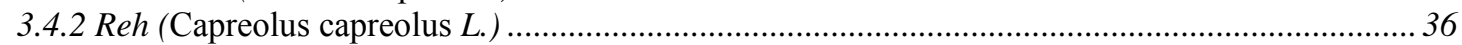

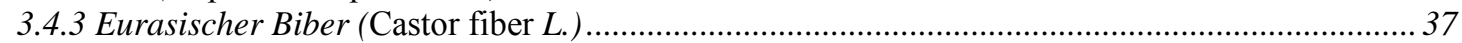

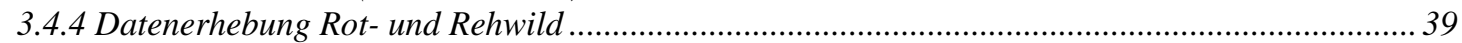

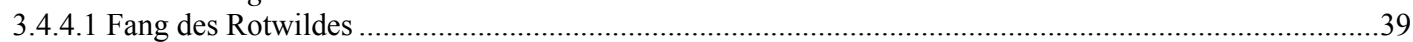

3.4.4.2 Fang des Rehwildes.............................................................................................................42

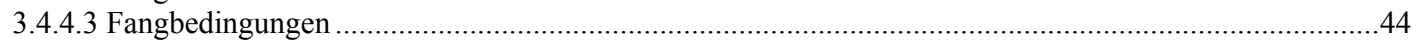

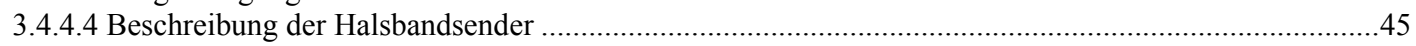

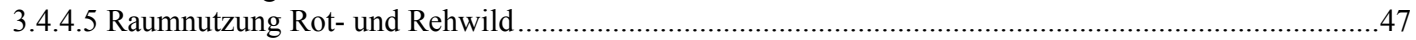

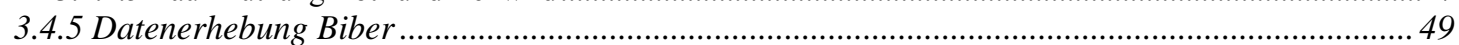

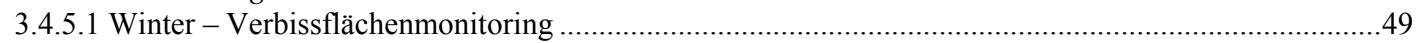

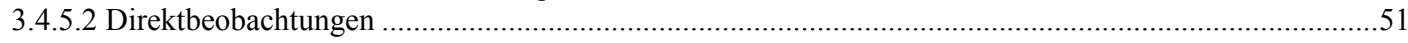

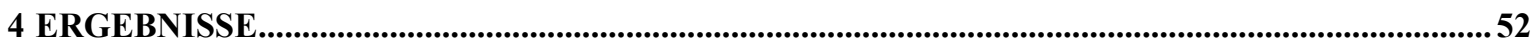

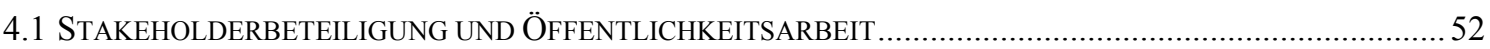

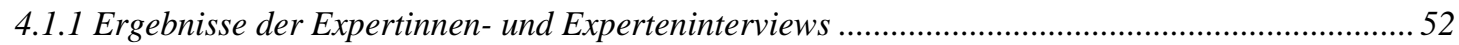

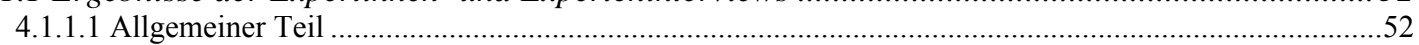

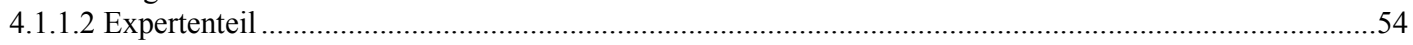

4.1.1.3 Die Lobau in 10 Jahren: Trends, Gefährdungen, Hoffnungen................................................................56

4.1.2 Diskussion der Ergebnisse im Workshop ........................................................................... 57 
4.1.3 Diskussion der Endergebnisse im AK Raumplanung und Workshop ..........................................60

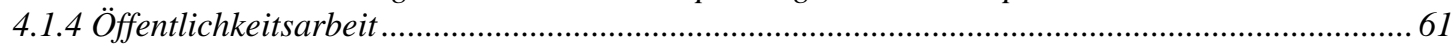

4.2 MEINUNG DER LOKALEN BEVÖLKERUNG UND DER BESUCHER UND BESUCHERINNEN .........................6 63

4.2.1 Ergebnisse der postalischen Befragung der lokalen Bevölkerung ............................................63

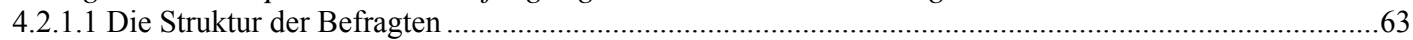

4.2.1.2 Bedeutung von Erholungs- und Naturschutzgebieten für den Wohnort und die Ortsverbundenheit .......63

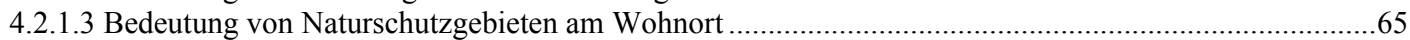

4.2.1.4 Bedeutung der Lobau als Erholungsraum und Verbundenheit mit ihr ..................................................65

4.2.1.5 Empfinden des Besuchsaufkommens und Ausweichverhalten...........................................................67

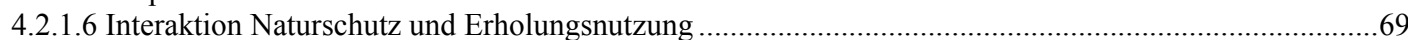

4.2.1.7 Der Nationalpark als touristisches Angebot …...................................................................................71

4.2.1.8 Zusammenhänge zwischen Ortsverbundenheit und den Konzepten der Erholungsforschung und

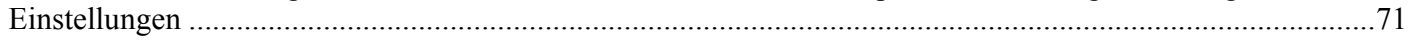

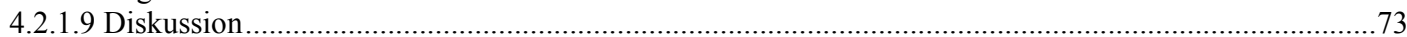

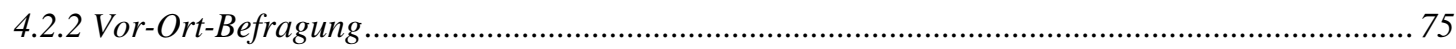

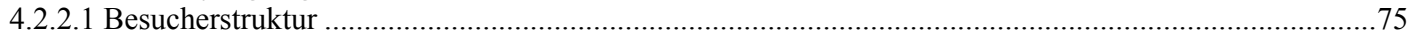

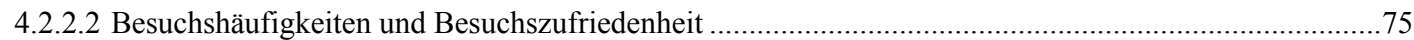

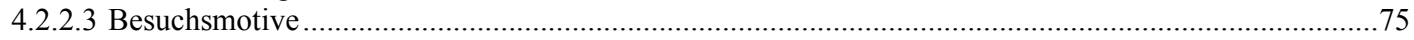

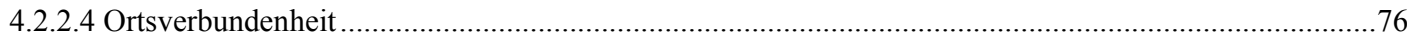

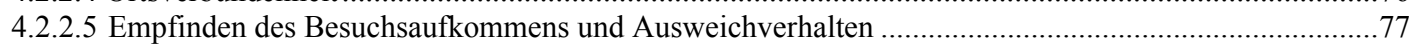

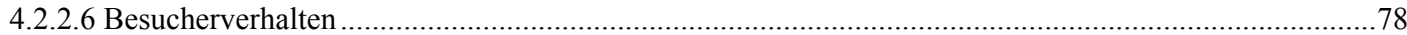

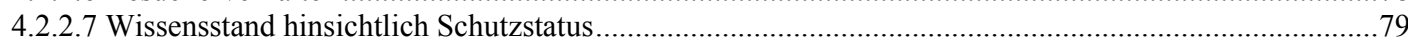

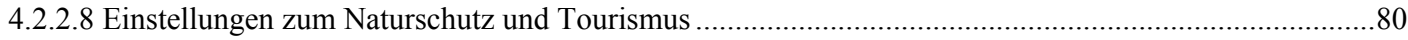

4.2.2.9 Zusammenhänge zwischen Ortsverbundenheit und den Konzepten der Erholungsforschung und

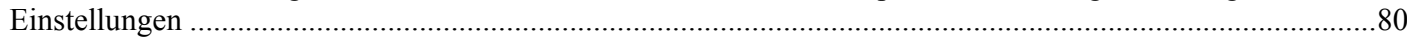

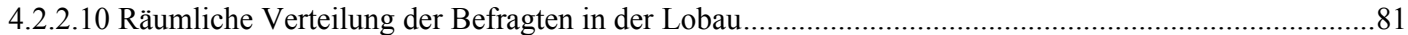

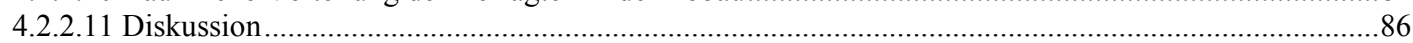

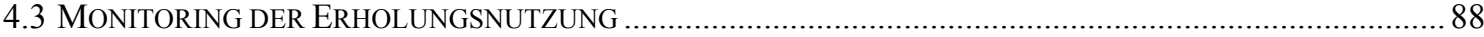

4.3.1 Ergebnisse der einjährigen bildgestützten Besucherzählung .................................................. 88

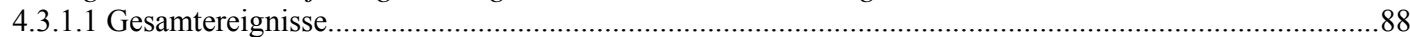

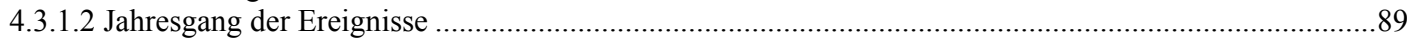

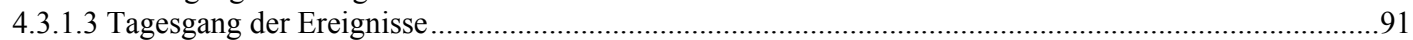

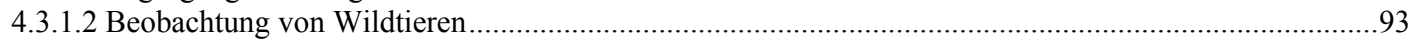

4.3.2 Vergleich der Besucherzahlen an den Standorten.................................................................. 94

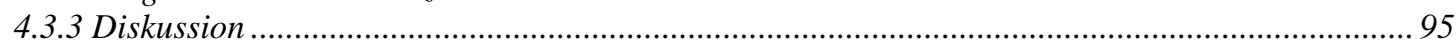

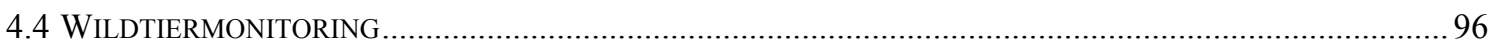

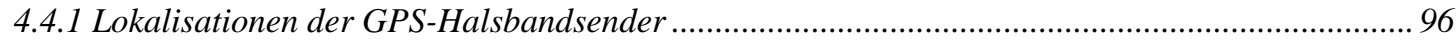

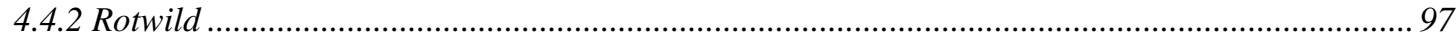

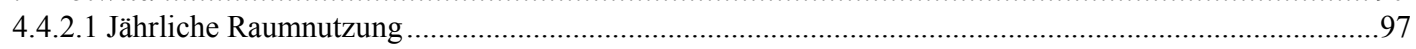

4.4.2.2 Sommer- und Winterkernstreifgebiet .........................................................................................99

4.4.2.3 Tag- und Nachtstreifgebiete während der Vegetationsperiode ..............................................................100

4.4.2.4 Kernstreifgebiete an besucherstarken und besucherschwachen Tagen..............................................101

4.4.2.5 Einfluss der Besuchernutzung auf die Raumnutzung des Rotwildes.................................................101

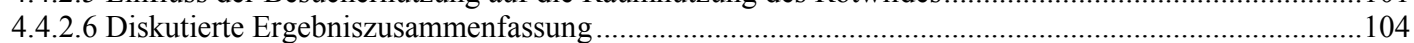

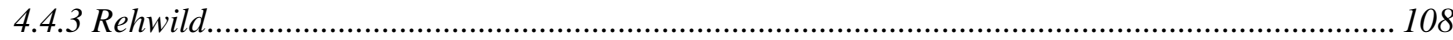

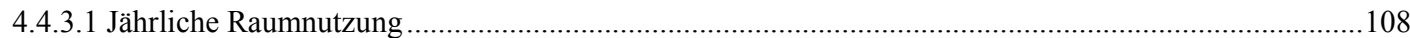

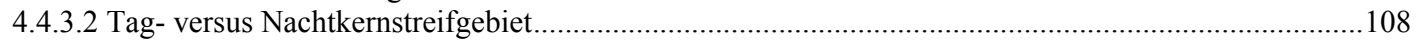

4.4.3.3 Raumnutzung an besucherstarken und besucherschwachen Tagen ..................................................110

4.4.3.4 Einfluss der Besuchernutzung auf die Raumnutzung von Rehwild.....................................................111

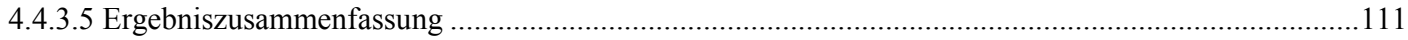

4.4.4 Eurasischer Biber (Castor fiber L.) ................................................................................. 113

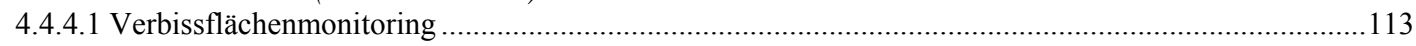

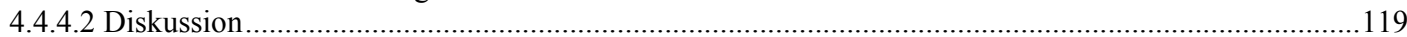

4.5 DISKUSSION DER DATEN UND AUSWIRKUNGEN DER ERHOLUNGSNUTZUNG AUF WILDTIERE..............120

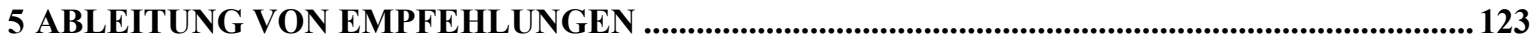

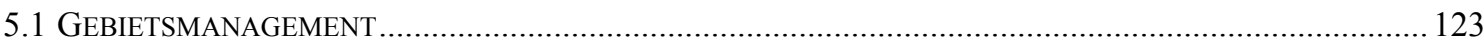

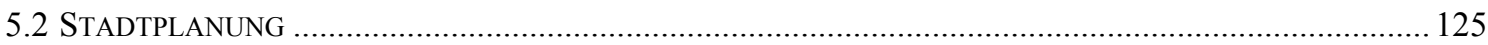

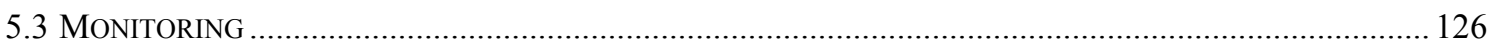

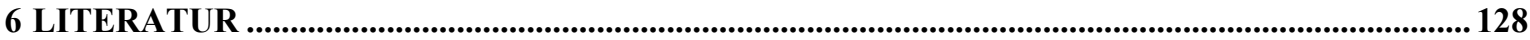




\section{Danksagung}

Bei den folgenden Institutionen und Personen möchten wir uns für die Unterstützung ganz herzlich bedanken:

Dem Forstamt der Stadt Wien, Magistratsabteilung 49, Nationalpark Forstverwaltung Lobau

Der Nationalpark Donau-Auen GmbH

Der MA22 Wien

BH Gänserndorf

Dipl.-Ing. Karolina Taczanowska

Dipl.-Ing. Thomas Meitz

Dipl.-Ing. Petra Sterl

Univ.Prof. Dr. Klaus Hackländer

Dr. Rosemarie Parz-Gollner

Dipl.-Ing. Mark Wöss

Mag. Elmar Gartlehner

Ao.Prof. Dr. Wolfgang Haider

Dr.med.vet. Wolfgang Zenker

Dipl. Tzt. Wilfried Laubichler

Dr.med.vet. Folko Balfanz

Frau Wolf (Gasthaus Staudigl)

Dem Befragungsteam des ILEN

Den befragten Bewohnerinnen und Bewohnern des Bezirks Donaustadt und der Gemeinde Groß-Enzersdorf

Den befragten Besucherinnen und Besuchern der Lobau 


\section{Kurzfassung}

Städtische Biosphärenparke sind durch hohe Besucherzahlen gekennzeichnet, womit sowohl die ökologische als auch die soziale Tragfähigkeit dieser Gebiete oft überschritten ist. Ziel des über drei Jahre laufenden Projekts war die Erfassung von Indikatoren der sozialen und ökologischen Tragfähigkeit des stark besuchten und flächenmäßig kleinen Biosphärenparks Untere Lobau in Wien als Beitrag zur Verbesserung des Gebietsmanagements.

Untersucht wurde dabei

(1) die Bedeutung der Lobau als Erholungsraum für die Anwohnenden als auch für die Besucher und Besucherinnen,

(2) der Einfluss von hohen Besucherfrequenzen auf das Erholungserlebnis von Besuchern und Besucherinnen und die daraus resultierenden Ausweichstrategien,

(3) der Einfluss der Erholungsnutzung auf die Wildtiere (Rotwild (Cervus elaphus), Rehwild (Capreolus capreolus), Biber (Castor fiber)).

Die Ergebnisse unter Beteiligung lokaler Akteure sollen zur Entwicklung von Empfehlungen für ein integratives und nachhaltiges Management und Monitoring des Biosphärenparks führen.

Ergebnis der Studie war, dass die Lobau für das Erholungsgeschehen eine einzigartige Rolle spielt. Die hohen Besucherzahlen führen allerdings zu einer Minderung der Qualität des Erholungserlebnisses und zu einem zeitlich-räumlichen Ausweichen, womit Ruhezeiten und Ruhezonen für die Wildtiere reduziert werden. Während die Auswirkungen der Erholungsnutzung auf den Biber aufgrund seiner Dämmerungs- und Nachtaktivität als eher vernachlässigbar einzustufen sind, zeigten die TelemetrieAufnahmen, dass Rot- und Rehwild auf die Erholungsnutzung reagieren. Sie meiden Wege mit hohen Besucherfrequenzen bzw. halten sich unter Tags versteckt.

Durch das geringe Bewusstsein der Befragten hinsichtlich ihrer eigenen Störwirkung - die Hälfte der Hunde ist nicht an der Leine - und durch die flächenhafte und intensive Nutzung der Unteren Lobau durch die Erholungsnutzung und durch den motorisierten Verkehr scheint sich ein Nationalparkeffekt nicht einzustellen.

Um eine nachhaltige Entwicklung des Biosphärenparks Untere Lobau zu garantieren, müssen Maßnahmen insbesondere im Umfeld des Biosphärenparks getätigt werden. Eine Ausgestaltung des Lobau-Vorlandes für die Erholungsnutzung sowie eine Ausdehnung des Biosphärenparks auf das Umland werden empfohlen. 